\title{
Novel noninvasive anthropometric measure in preterm and full-term infants: normative values for waist circumference:length ratio at birth
}

\author{
Alexander Holston ${ }^{1}$, Theophil Stokes ${ }^{2}$, Cara Olsen ${ }^{3}$, Y. Sammy Choi ${ }^{4,5}$, Jerri Curtis ${ }^{2}$, Jason Higginson², Chinenye Adimora ${ }^{6}$ and \\ Carl E. Hunt ${ }^{2}$
}

BACKGROUND: Waist circumference:length ratio (WLR) and ponderal index (PI) appear to be useful markers of visceral and total adiposity, respectively. However, there are no normative birth data across the full range of gestational ages.

METHODS: In this retrospective cohort study of 500 preterm and 1,426 full-term infants, born in 1998 and 2008 at three military hospitals, the percentile growth curves for WLR and $\mathrm{PI}$ were calculated. There were no sex differences, and results were combined to obtain values from 26 to 42 wk gestation.

RESULTS: Between 26 and 42 wk gestation, median birth WLR increased from 0.55 to 0.62 , and median PI increased from 21.1 to 25.6. The adjusted mean WLR at birth among infants born $<34$ wk increased from 0.55 in 1998 to 0.58 in 2008 ( $P=0.048$ ), suggesting that early-preterm infants born in 2008 had greater abdominal adiposity than those born in 1998.

CONCLUSION: We report normative birth data for WLR and PI in preterm and full-term infants by gestational age and sex. WLR and PI may be useful as clinical markers of visceral and overall adiposity. In conjunction with other anthropometric measures, WLR and PI may be useful to monitor postnatal nutrition and growth and assess risk for later obesity and cardiometabolic disorders.

M any studies have shown that excess body adiposity, especially visceral adiposity, increases the risk for later cardiometabolic disorders. Measuring the waist circumference (WC) (1) has been used in adults, adolescents, and children as a simple and inexpensive population-based strategy for a clinical practice-based estimate of visceral adiposity (1). Elevated WC was associated with diabetes, coronary artery disease, morbidity, and mortality in adult North American, Asian, and European populations (2-4). WC has been shown to correlate with the risk of type 2 diabetes, in some cases better than weight, height, body mass index (BMI), or skinfold thickness (5). The ratio of standing height to $\mathrm{WC}$ has been utilized in some studies to normalize for age, sex, and race/ethnicity. The waist circumference:height ratio (WHtR) was closely associated with coronary risks such as hypertension, hyperglycemia, hypertriglyceridemia, hypercholesterolemia, and lower levels of high-density lipoprotein (3). WHtR was a better predictor of metabolic syndrome than BMI in Mexican children aged 6-12 y (6). Data for infants, however, are lacking. There are some very limited population-based norms in infants born full term for WC and waist circumference:length ratio (WLR) based on supine length, but there are no birth data in infants born preterm $(7,8)$.

In current practice, neonatologists aim to achieve postnatal growth rates comparable to intrauterine growth based on estimated gestational age (EGA) and weight (9). But weight by itself tells only a little about the overall body composition. The BMI has been used to evaluate growth and as a surrogate indicator of obesity. However, the BMI has not been utilized in infants, and it does not reliably distinguish between growth in lean and that in fat mass. Some investigators have, thus, concluded it is time to move beyond BMI as a marker of obesity and focus on more direct measures of body composition (10). Usefulness of the ponderal index (PI) in assessing fat mass is unclear, but it has been reported to be an indirect measure of soft tissue mass and to correlate well with total skinfold thickness (11). Although WLR and PI have not been used in routine neonatal clinical practice, these two measurements have the potential advantage of assessing visceral and total fat mass, respectively, more directly than achievable with weight alone or even BMI.

Our underlying hypothesis for this retrospective chart review was that (i) visceral adiposity is a useful measure for tracking risk for cardiometabolic sequelae in neonates, including infants born preterm, and (ii) WC is a useful marker of visceral adiposity, in particular when normalized for body length. On the basis of birth data from three medical centers in this retrospective cohort study, we have previously reported that

'Department of Pediatrics, Naval Medical Center Portsmouth, Portsmouth, Virginia; ${ }^{2}$ Department of Pediatrics, Walter Reed National Military Medical Center and Uniformed Services University of the Health Sciences, Bethesda, Maryland; ${ }^{3}$ Biostatistics Consulting Center, Uniformed Services University of the Health Sciences, Bethesda, Maryland; ${ }^{4}$ Department of Medicine, Womack Army Medical Center, Fort Bragg, North Carolina; ${ }^{5}$ Department of Pediatrics, Womack Army Medical Center, Fort Bragg, North Carolina; ${ }^{6}$ Department of Obstetrics and Gynecology, Walter Reed National Military Medical Center and Uniformed Services University of the Health Sciences, Bethesda, Maryland. Correspondence: Carl E. Hunt (carl.hunt@usuhs.edu) 
preterm infants have disproportionate increases in WLR and PI at term-equivalent age, suggestive of increased visceral and total adiposity, respectively (12). Although these data suggest that WLR and PI may have clinical utility as simple and inexpensive indicators of visceral and total fat mass, respectively, there are no available normative birth data across the full range of gestational ages. The purpose of this report is to establish normative values for WLR and PI in preterm and full-term infants across the full range of EGA at birth from 26 to $42 \mathrm{wk}$. We also examine two epochs a decade apart to determine whether, in association with the obesity epidemic, infants born in 2008 have a greater apparent total adiposity and visceral adiposity than infants born in 1998 and are therefore potentially at greater risk for later cardiometabolic disorders.

\section{RESULTS}

Our initial database included 522 singleton preterm infants and 1,438 singleton full-term infants. After ineligible infants and outliers were identified and corrected or removed from the data set, our final database for analysis included 500 preterm and 1,426 full-term infants. This included 270 and 230 preterm infants in 1998 and 2008, respectively, and 753 and 673 full-term infants in 1998 and 2008, respectively.

The maternal demographics of women who delivered preterm and full term are presented in Table 1. Prepregnancy weight was significantly higher among women who delivered in 2008 for preterm $(P=0.042)$ and for full-term births $(P=0.026)$. Prepregnancy BMI, however, was significantly increased in 2008 only for women who delivered full-term $(P=0.0036)$. Despite this increase, weight gain during pregnancy was similar in the two study periods for all women. Women who delivered preterm in 2008 were more likely to be diagnosed with gestational hypertension $(P=0.029)$. The 1 -h glucose level was increased in $2008(P=0.0045)$, but only for women delivering a full-term infant.

Among all infants born preterm, adjusted significance testing identified a significant increase in birth weight in the 2008 epoch $(P=0.01)$, but not in the $Z$ score of the birth weight (Table 2). There was no difference between the two epochs for adjusted length, head circumference, and WC. There were also no significant adjusted differences among preterm infants between the two epochs for BMI, PI, or WLR. In a

Table 1. Maternal demographics for 1998 and 2008 birth cohorts

\begin{tabular}{|c|c|c|c|c|c|c|}
\hline & \multicolumn{3}{|c|}{ Preterm (<37 wk) } & \multicolumn{3}{|c|}{ Full-term ( $\geq 37$ wk) } \\
\hline & 1998 & 2008 & $P$ value & 1998 & 2008 & $P$ value \\
\hline$N$ & 270 & 230 & & 753 & 673 & \\
\hline Age (y) & $27.7 \pm 5.8$ & $28.4 \pm 6.2$ & 0.22 & $27.7 \pm 5.7$ & $28.4 \pm 6.2$ & 0.50 \\
\hline Pre-pregnancy weight (lbs) & $142 \pm 32$ & $153 \pm 37$ & 0.042 & $146 \pm 31$ & $151 \pm 33$ & 0.026 \\
\hline Weight gain (lbs) & $29.5 \pm 14.8$ & $29.2 \pm 16.5$ & 0.87 & $33.2 \pm 11.8$ & $33.4 \pm 14.7$ & 0.83 \\
\hline Prepregnancy BMI $\left(\mathrm{kg} / \mathrm{m}^{2}\right)$ & $24.7 \pm 5.8$ & $25.8 \pm 6.1$ & 0.25 & $24.3 \pm 5$ & $25.4 \pm 5.3$ & 0.0036 \\
\hline Race $(n, \%)$ & & & 0.040 & & & $<0.0001$ \\
\hline Caucasian & $124(45.9 \%)$ & $108(47 \%)$ & & $435(57.8 \%)$ & $399(59.3 \%)$ & \\
\hline African-American & $53(19.6 \%)$ & 48 (20.9\%) & & $99(13.1 \%)$ & $102(15.2 \%)$ & \\
\hline Hispanic & $7(2.6 \%)$ & $15(6.5 \%)$ & & $46(6.1 \%)$ & $31(4.6 \%)$ & \\
\hline Asian & $8(3 \%)$ & $13(5.7 \%)$ & & $31(4.1 \%)$ & $26(3.9 \%)$ & \\
\hline Hawaiian/Pacific Islander & $22(8.1 \%)$ & $9(3.9 \%)$ & & $57(7.6 \%)$ & $11(1.6 \%)$ & \\
\hline Other/unknown & $56(20.7 \%)$ & $37(16.1 \%)$ & & $85(11.3 \%)$ & $104(15.5 \%)$ & \\
\hline Gestational hypertension $(N, \%)$ & $6(4.4 \%)$ & $18(11.3 \%)$ & 0.029 & $10(2.9 \%)$ & $18(3.7 \%)$ & 0.54 \\
\hline Preeclampsia $(n, \%)$ & $34(12.6 \%)$ & $32(13.9 \%)$ & 0.66 & $14(1.9 \%)$ & $23(3.4 \%)$ & 0.065 \\
\hline Gestational diabetes $(n, \%)$ & $17(6.3 \%)$ & $23(10 \%)$ & 0.13 & $41(5.4 \%)$ & $23(3.4 \%)$ & 0.065 \\
\hline 1-h glucose test (mg/dl) & $117 \pm 39$ & $118 \pm 26$ & 0.89 & $108 \pm 27$ & $115 \pm 27.2$ & 0.0045 \\
\hline Education ( $n, \%)$ & & & 0.21 & & & 0.90 \\
\hline High school graduate or less & $45(38.1 \%)$ & $61(31.3 \%)$ & & $117(36 \%)$ & $134(35.5 \%)$ & \\
\hline Some college or more & $73(61.9 \%)$ & $134(68.7 \%)$ & & $208(64 \%)$ & $243(64.5 \%)$ & \\
\hline Rank of active duty member $(n, \%)$ & & & 0.51 & & & 0.74 \\
\hline Enlisted & $145(74.4 \%)$ & $88(71 \%)$ & & $476(63.2 \%)$ & $320(72.7 \%)$ & \\
\hline Officer $^{\mathrm{a}}$ & $50(25.6 \%)$ & $36(29 \%)$ & & $187(24.8 \%)$ & $120(27.3 \%)$ & \\
\hline
\end{tabular}

Data are presented as means \pm SD or as N (\%). The following sample sizes were used to calculate means for women delivering preterm in 1998 and 2008 and full term in 1998 and 2008 : pregravid weight was available for 75, 100, 317, and 456 women, respectively; weight gain was available for 70, 100, 309, and 456 women, respectively; pregravid BMI was available for 74, 96, 314, and 440 women; 1-h glucose was available for 60, 86, 191, and 385 women, respectively. Percentages were calculated based on the available sample size and not as a percentage of the entire population. The diagnosis of gestational hypertension was available for 137, 159, 340, and 483 women, respectively. Values in bold indicate statistically significant differences $(P<0.05)$.

anank as officer requires college degree. 


\section{Birth waist circumference:length ratio}

Table 2. Infant body size and anthropometric measures at birth for 1998 and 2008 for boys and girls combined

\begin{tabular}{|c|c|c|c|c|c|c|}
\hline & \multicolumn{3}{|c|}{ Preterm $(<37 w k)$} & \multicolumn{3}{|c|}{ Full-term ( $\geq 37$ wk) } \\
\hline & 1998 & 2008 & $P$ value & 1998 & 2008 & $P$ value \\
\hline Estimated gestational age (wk) & $34.5 \pm 0.74$ & $34.8 \pm 0.6$ & 0.45 & $38.7 \pm 0.2$ & $38.6 \pm 0.2$ & 0.24 \\
\hline Birth weight (kg) & $2.47 \pm 0.11$ & $2.31 \pm 0.10$ & 0.01 & $3.50 \pm 0.07$ & $3.38 \pm 0.07$ & 0.01 \\
\hline Birth weight ( $Z$ score) & $0.43 \pm 0.12$ & $0.31 \pm 0.11$ & 0.4 & $0.12 \pm 0.05$ & $0.08 \pm 0.05$ & 0.53 \\
\hline Length ( $Z$ score) & $0.42 \pm 0.13$ & $0.41 \pm 0.11$ & 0.91 & $0.18 \pm 0.05$ & $0.41 \pm 0.05$ & $<0.001$ \\
\hline Head circumference $(\mathrm{cm})$ & $31.3 \pm 0.45$ & $31.0 \pm 0.39$ & 0.34 & $34.7 \pm 0.25$ & $34.1 \pm 0.23$ & $<0.001$ \\
\hline Head circumference ( $Z$ score) & $0.24 \pm 0.14$ & $0.27 \pm 0.12$ & 0.77 & $0.15 \pm 0.04$ & $0.06 \pm 0.05$ & 0.12 \\
\hline Waist circumference (cm) & $26.5 \pm 0.62$ & $26.7 \pm 0.53$ & 0.55 & $31.0 \pm 0.38$ & $31.0 \pm 0.35$ & 0.94 \\
\hline $\mathrm{BMI}\left(\mathrm{kg} / \mathrm{m}^{2}\right)$ & $11.7 \pm 0.53$ & $11.2 \pm 0.46$ & 0.096 & $13.5 \pm 0.22$ & $12.9 \pm 0.21$ & $<0.001$ \\
\hline
\end{tabular}

Data are presented as adjusted means \pm SEM or as $n$ (\%). BMI and PI were calculated for all infants with a recorded weight and length. $Z$ scores for weight, length, and head circumference were adjusted for delivery site and race. Adjusted $P$ values for weight, length, head circumference, waist circumference, BMI, PI, and WLR controlled for delivery site, sex, and estimated gestational age, and for maternal race, pregravid BMI, gestational weight gain, preeclampsia, and gestational diabetes. The following sample sizes were used to calculate means for infants in this table: birth weight and gestational age were available for 434 preterm and 1,339 term infants; length, BMI, and PI were available for 417 preterm and 1,320 term infants; head circumference for 416 preterm and 1,019 term infants; waist circumference for 279 preterm and 789 term infants; and WLR for 272 preterm and 786 term infants. Values in bold indicate statistically significant differences $(P<0.05)$.

$\mathrm{Pl}$, ponderal index; WLR, waist circumference:length ratio.

planned subgroup analysis of early- and late-preterm infants, there were no differences in the late-preterm (34-36 wk) subgroup between the two epochs (Table 3 ). In the early-preterm $(<34 \mathrm{wk})$ subgroup, there was a significant decrease in birth weight and length $(P=0.02$ and $P=0.002$, respectively), but the $Z$ score for the birth weight did not differ. Moreover, the WLR was increased in the 2008 epoch in early-preterm infants $(P=0.048)$.

Among infants born full-term, unlike reported national trends, infants born in 2008 were not delivered earlier than in 1998 (Table 2) (13). When comparing differences in birth anthropometrics for full-term infants born in 2008 to those born in 1998, adjusted comparison showed statistically significant decreases in birth weight $(P=0.01)$ and head circumference $(P<0.001)$, but the $Z$ scores for weight and head circumference did not differ. Birth length and length $Z$ score were significantly increased in the 2008 epoch $(P=0.04$ and $P$ $<0.001$, respectively), whereas BMI and PI were significantly decreased in 2008 as compared with 1998 ( $P<0.001$ for each). The WC and WLR were similar in the two epochs.

Because none of the absolute overall differences between the 1998 and 2008 cohorts was clinically significant, we combined the two cohorts to calculate the birth percentiles for WLR and PI for each EGA week at birth. A total of 550 males and 508 females had a WC and length at birth to calculate the WLR, and 999 males and 831 females had a birth weight and length to calculate the PI (Table 4). There were insufficient data to include infants $<30 \mathrm{wk}$ at birth when calculating percentiles separately for males and females.

For the least mean square (LMS) calculations, models with a high degree of smoothing tended to fit the data equally well as compared with more complex models, and the smooth models were preferentially selected because of the small sample size in some EGA categories. $Z$ scores were calculated for each infant in the sample using the estimated LMS values. The distribution of these $Z$ scores was approximately normal, with a mean of 0 and an SD of 1 for boys and girls for both WLR and PI. The results are presented separately for males and females for WLR (Figure 1) and for PI (Figure 2). The growth curves for boys and girls were compared using quantile regression. No statistically significant differences between boys and girls were observed at any quantile with respect to the main effect of sex, interaction with age, or interaction with age squared (all $P$ values $>0.05$, data not shown). We therefore combined male and female percentiles for WLR (Figure 1c) and PI (Figure 2c). When combined, the sample size permitted an EGA range of 26-42 wk for both WLR and PI. Median WLR (Figure 1c) increased from 0.55 at $26 \mathrm{wk}$ to 0.62 at $42 \mathrm{wk}$ EGA. Median PI increased from $21.1 \mathrm{~kg} / \mathrm{m}^{3}$ at $26 \mathrm{wk}$ to 25.6 at $42 \mathrm{wk}$ EGA.

\section{DISCUSSION}

We report the first normative data for WLR and PI at birth in preterm and full-term infants by sex and week of EGA at birth across an EGA range of 26-42 wk (Figures 1 and 2). These birth data for WLR using a simple and inexpensive noninvasive measure suggest that preterm infants born $<34 \mathrm{wk}$ in 2008 had greater abdominal adiposity (Table 3 ) than infants from similar EGA births in 1998. This increase was not observed in late-preterm infants. WLR and PI were similar in male and female births (Figures 1 and 2), and the combined results for WLR and PI enabled us to calculate percentiles for infants born as early as $26 \mathrm{wk}$ instead of just $30 \mathrm{wk}$. The progressive increases in WLR and PI with increasing EGA at birth were consistent with progressive increases in visceral and total fat 


\section{Articles $\mid$ Holston et al.}

Table 3. Early- and late-preterm body size and anthropometric measures at birth from 1998 and 2008 for boys and girls combined

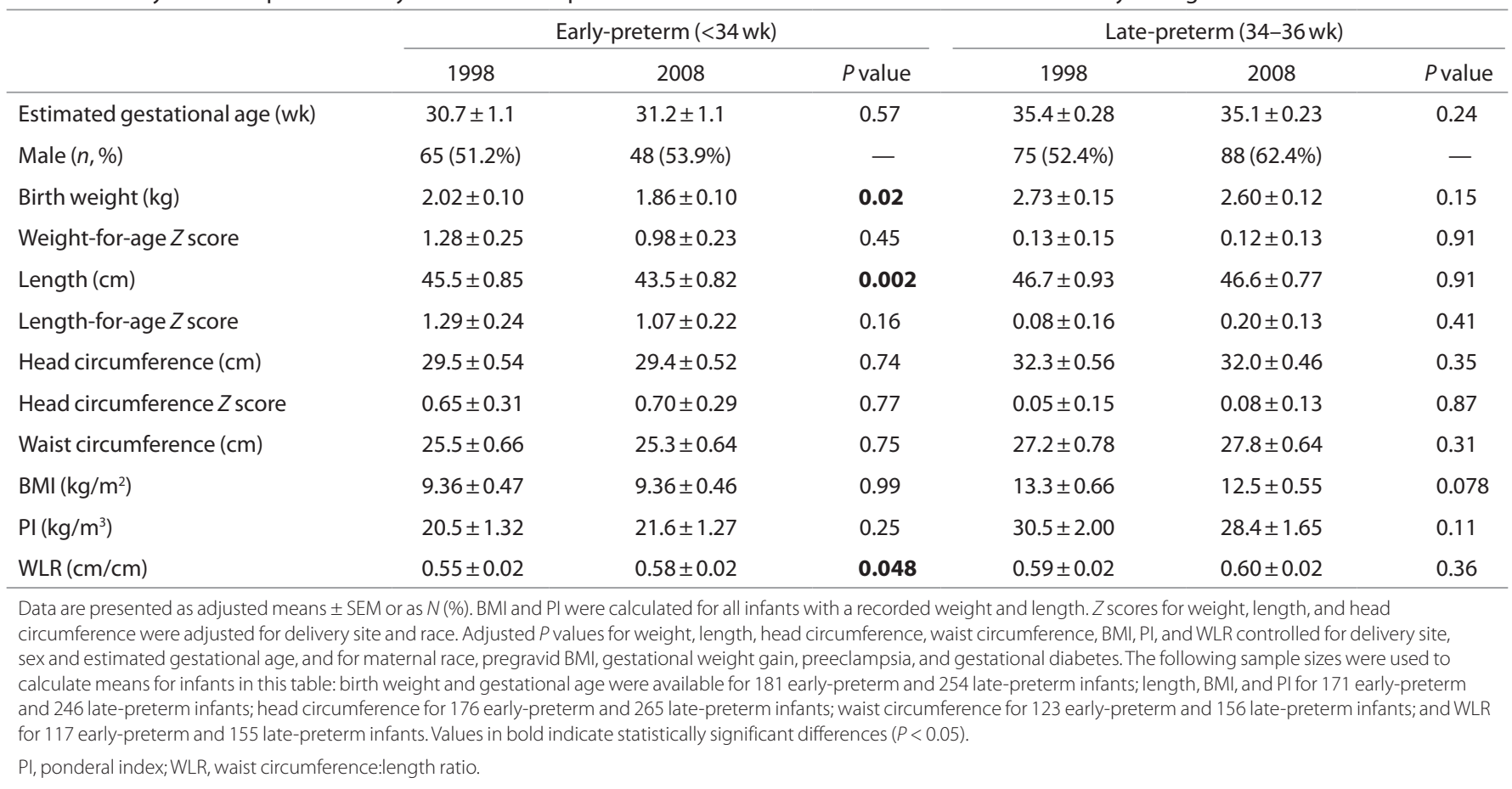

mass, respectively. The impact of greater visceral adiposity at birth in $<34$ wk EGA infants in 2008 on risk for later cardiometabolic sequelae is unknown. However, these normative birth data for WLR and PI may provide a useful baseline based on EGA for routine clinical tracking of comprehensive anthropometric measures as related to neonatal and infant nutrition and to risk for cardiometabolic sequelae.

Fetal growth and birth anthropometrics have historically focused on weight, length, and head circumference for routine clinical monitoring (14). However, weight gain by itself is a poor indicator of body composition and hence of optimum nutrition (15). Only limited data are available regarding body composition at birth, especially as based on EGA at birth and across the full range of EGA occurring today. Although BMI has often been used as a surrogate for adiposity at later ages, it has often not reliably quantified total fat mass in either pediatric or adult populations and hence can provide insufficient information about body fat mass or percentage body fat $(16,17)$.

Several direct measures of body composition have been developed for research purposes. Dual energy X-ray absorptiometry imaging has been used as a measure of total body fat and has been shown in adults to correlate with WHtR $(18,19)$. Air displacement plethysmography is an innovative, accurate, and precise way to assess fat mass in infants and to track changing body composition, even in preterm infants (20-22). However, neither of these methods quantifies visceral fat mass, which is a significant limitation given the metabolic activity and importance of this compartment (18,23). Other investigators have used whole-body magnetic resonance imaging, total-body electric conductivity, or ultrasound to evaluate overall adiposity or fat mass and visceral fat mass $(18,24,25)$. Use of these various direct measures of body fat mass and, in particular, visceral adiposity, is important in research studies and for validation of simpler and inexpensive measures such as WLR and PI, but they are not practical for routine clinical use or in population-based studies.

Adverse cardiometabolic outcomes appear to be more directly related to visceral adiposity than total fat mass, and visceral adiposity has been identified as an important predictor of later development of metabolic syndrome and cardiovascular sequelae including individuals born preterm (4,26-28). WC has been confirmed to be a valid measure of visceral obesity in adolescents and adults across the age span and in varying racial/ethnic groups $(16,29,30)$. WC varies according to sex, age, and race (5). However, normalizing WC for length or height by calculating WLR or WHtR, respectively, eliminates age, sex, and racial/ethnic differences $(3,6,31,32)$.

Normative WC values have been reported in children $\geq 2 \mathrm{y}$ of age and in adolescents of various ethnicities $(1,7,33,34)$. However, studies of WC in newborn infants are limited. One study from Sweden included an unspecified number of latepreterm infants but did not report separate results for infants born preterm and full term (7). Their median WC values for boys and girls of 35.7 and $35.0 \mathrm{~cm}$, respectively, are higher than our full-term WC values (Table 2). Newborn males had a median WLR of 0.69 , and newborn females had a median WLR of 0.687 that is higher than our mean values (Table 2) (7). Our data, however, are similar to WC data published in an American study that reported a mean value of $31 \mathrm{~cm}$ for boys and girls combined (8).

The PI is not commonly used in practice today. Its use as a measure of adiposity is unclear (35). PI has been demonstrated to correlate with the total percentage of body fat in infants as measured by skinfold thickness and air displacement 
Table 4. Percentile cutoff values for WLR and the PI

\begin{tabular}{|c|c|c|c|c|c|c|c|c|c|c|c|c|c|c|c|c|}
\hline \multirow{2}{*}{$\begin{array}{l}\text { EGA } \\
\text { (wk) }\end{array}$} & \multicolumn{8}{|c|}{ Males } & \multicolumn{8}{|c|}{ Females } \\
\hline & $N$ & 2nd & 10th & 25th & 50th & 75th & 90th & 98th & $N$ & 2nd & 10th & 25th & 50th & 75th & 90th & 98th \\
\hline \multicolumn{17}{|c|}{ WLR (cm/cm) } \\
\hline 31 & 4 & 0.50 & 0.52 & 0.55 & 0.58 & 0.61 & 0.64 & 0.68 & 10 & 0.49 & 0.52 & 0.54 & 0.56 & 0.60 & 0.63 & 0.70 \\
\hline 32 & 20 & 0.49 & 0.52 & 0.55 & 0.58 & 0.61 & 0.64 & 0.68 & 12 & 0.50 & 0.52 & 0.54 & 0.57 & 0.60 & 0.64 & 0.70 \\
\hline 34 & 27 & 0.49 & 0.53 & 0.55 & 0.59 & 0.62 & 0.65 & 0.68 & 29 & 0.51 & 0.54 & 0.56 & 0.59 & 0.62 & 0.65 & 0.70 \\
\hline 35 & 30 & 0.50 & 0.53 & 0.56 & 0.59 & 0.62 & 0.65 & 0.69 & 13 & 0.52 & 0.55 & 0.57 & 0.60 & 0.63 & 0.66 & 0.70 \\
\hline 36 & 37 & 0.50 & 0.54 & 0.57 & 0.60 & 0.63 & 0.66 & 0.70 & 27 & 0.53 & 0.55 & 0.58 & 0.60 & 0.63 & 0.66 & 0.70 \\
\hline 37 & 44 & 0.51 & 0.55 & 0.58 & 0.61 & 0.64 & 0.67 & 0.70 & 33 & 0.53 & 0.56 & 0.58 & 0.61 & 0.64 & 0.67 & 0.70 \\
\hline 38 & 78 & 0.52 & 0.55 & 0.58 & 0.61 & 0.64 & 0.67 & 0.71 & 66 & 0.53 & 0.56 & 0.59 & 0.61 & 0.64 & 0.67 & 0.71 \\
\hline 42 & 5 & 0.53 & 0.56 & 0.59 & 0.62 & 0.65 & 0.67 & 0.71 & 7 & 0.54 & 0.58 & 0.60 & 0.63 & 0.65 & 0.68 & 0.70 \\
\hline \multicolumn{17}{|c|}{$\mathrm{PI}\left(\mathrm{kg} / \mathrm{m}^{3}\right)$} \\
\hline 30 & 18 & 16.81 & 18.38 & 19.79 & 21.58 & 23.65 & 25.82 & 29.11 & 12 & 16.17 & 17.78 & 19.17 & 20.84 & 22.67 & 24.45 & 26.93 \\
\hline 31 & 10 & 17.10 & 18.71 & 20.16 & 22.01 & 24.15 & 26.40 & 29.81 & 15 & 16.74 & 18.41 & 19.85 & 21.58 & 23.47 & 25.31 & 27.88 \\
\hline 32 & 33 & 17.38 & 19.04 & 20.53 & 22.44 & 24.65 & 26.98 & 30.51 & 20 & 17.29 & 19.02 & 20.50 & 22.29 & 24.24 & 26.15 & 28.80 \\
\hline 33 & 26 & 17.65 & 19.34 & 20.88 & 22.84 & 25.12 & 27.52 & 31.18 & 27 & 17.79 & 19.57 & 21.10 & 22.94 & 24.95 & 26.91 & 29.63 \\
\hline 34 & 47 & 17.94 & 19.68 & 21.25 & 23.26 & 25.61 & 28.08 & 31.85 & 48 & 18.23 & 20.06 & 21.62 & 23.51 & 25.57 & 27.58 & 30.37 \\
\hline 35 & 47 & 18.30 & 20.08 & 21.68 & 23.73 & 26.12 & 28.64 & 32.48 & 23 & 18.62 & 20.48 & 22.08 & 24.00 & 26.11 & 28.16 & 31.01 \\
\hline 42 & 13 & 20.79 & 22.38 & 23.78 & 25.50 & 27.45 & 29.42 & 32.30 & 8 & 20.00 & 22.01 & 23.72 & 25.79 & 28.05 & 30.26 & 33.32 \\
\hline
\end{tabular}

Data are presented for males and females for combined 1998 and 2008 birth cohorts for each week of estimated gestational age at birth from 30 to 42 wk. The 50 th percentile corresponds to the unadjusted median. These estimated gestational age- and sex-specific percentiles are obtained from the estimated $L, M$, and $S$ values using the least mean square method of Cole and Green (16).

EGA, estimated gestational age; PI, ponderal index; WLR, waist circumference:length ratio.

plethysmography $(11,36)$. Newborn data for PI are available for Chinese and German infants, but data in the United States are limited, especially for infants born preterm $(11,37)$. The most recent normative data for US preterm infants were published by Lubchenco et al. (38) in 1966 and only included Caucasian infants. Our PI data are similar among more premature infants and show lower cutoffs for late-preterm and term infants at the 90th percentile. This is similar to a recent US study that compared birth weight and PI for neonates born at $26-29 \mathrm{wk}$ gestation and concluded that the Lubchenco cutoffs were higher for both birth weight and PI (10). There is a need for simple and noninvasive methods to assess body composition in neonates (35). The availability of WLR and PI normative values at birth may help clinicians by providing an indirect baseline for evaluating neonatal nutritional intervention strategies, for comprehensive tracking of visceral and overall adiposity, and for assessing risk for later cardiometabolic sequelae. In relation to risk for later cardiometabolic sequelae, however, the optimum neonatal targets for WLR or PI remain unknown.

The primary strengths of our study include a large number of both preterm and full-term infants included from three geographic locations for two separate time periods $10 \mathrm{y}$ apart, analysis of results based on EGA at birth, and the availability of WC measurements from two sites. There are, however, several limitations. First, we do not have any direct measure of body composition to determine the extent of correlation of WLR and PI with direct measures of visceral fat mass and total body fat mass. Of particular note, however, WC has been confirmed to be a valid measure of visceral obesity in children, adolescents, and adults across the age span and in varying racial/ 
a

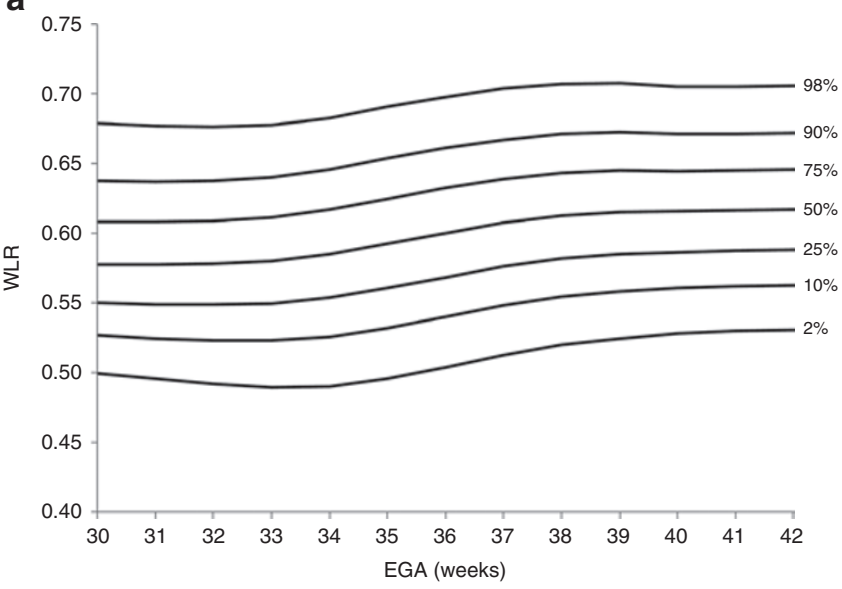

b

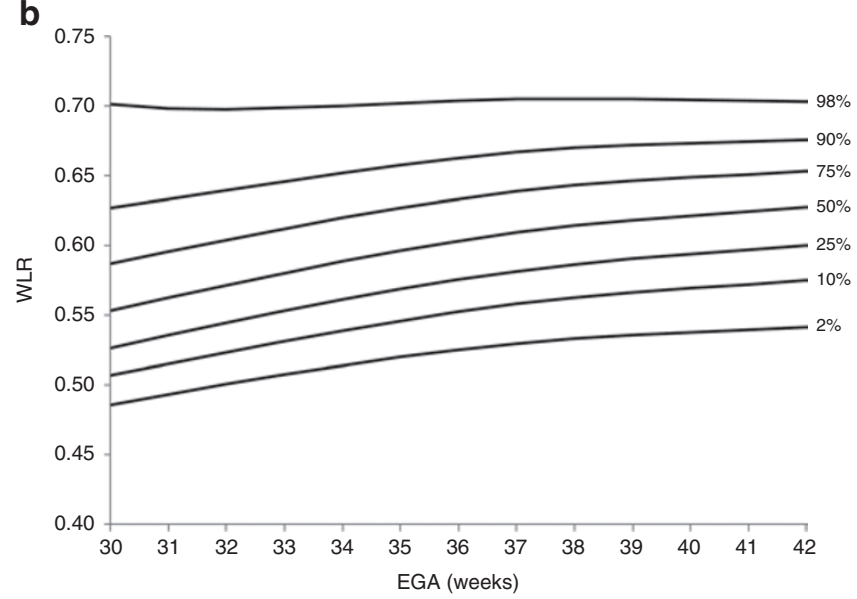

C

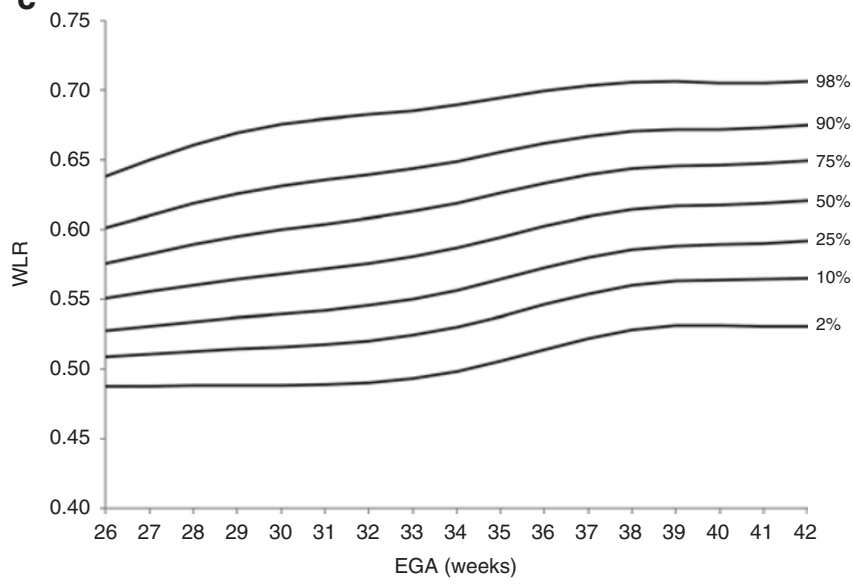

Figure 1. Smoothed percentile growth curves for WLR for EGAs at birth for (a) male infants born at 30-42 wk, (b) female infants born at 30-42 wk, and (c) both sexes combined born at 26-42 wk. Absolute values are presented in Table 4. EGA, estimated gestational age; WLR, waist circumference:length ratio.

ethnic groups, and normalizing WC for height by calculating waist-to-height ratio eliminates age, sex, and racial/ethnic differences $(3,6,16,29-32)$. Although we do have broad racial/ ethnic diversity, our families are not fully representative of the US population in that most parents are married, all have health insurance, most mothers have at least a high school education,
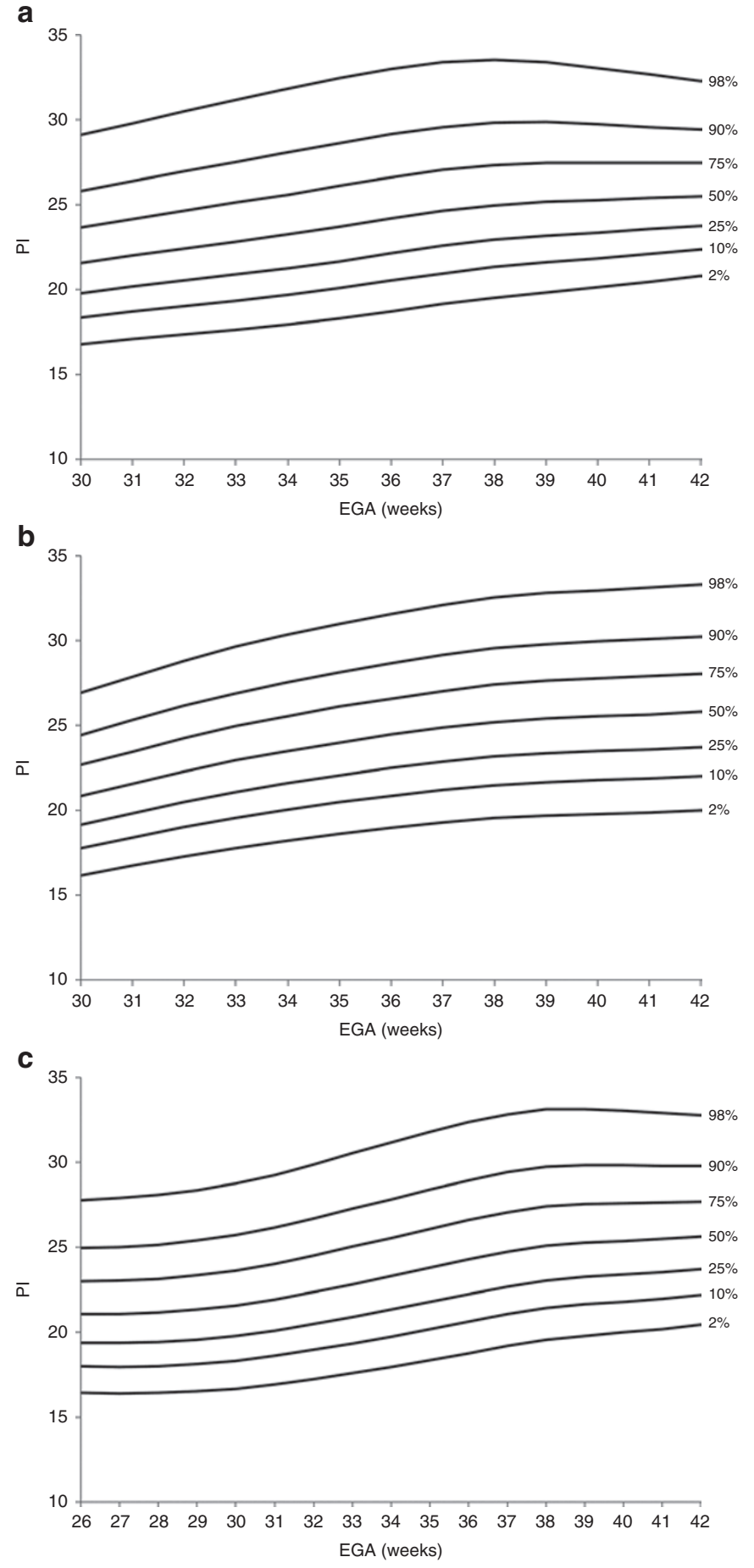

Figure 2. Smoothed percentile growth curves for PI for EGAs at birth for (a) male infants born at 30-42 wk, (b) female infants born at 30-42 wk, and (c) both sexes combined born at 26-42 wk. Absolute values are presented in Table 4. EGA, estimated gestational age; $\mathrm{Pl}$, ponderal index.

and self-reported fetal exposure is low for smoking and almost absent for alcohol and street drugs. Hence, our results may not be fully generalizable to the entire US population.

There are also inherent limitations to our retrospective chart review design. These include missing data, data entry errors, no standardized definitions for recorded maternal diagnoses, 
and lack of standardization of measurement methods, in particular for length and WC (12). Because WC data were collected at only two of our three study sites, we are only able to calculate WLR in a subset of infants. Despite smaller numbers, however, the statistical significance of these findings appears robust, and the results are consistent with our PI results based on a much larger sample size. Finally, as with any retrospective study design, our results are hypothesis generating and hence must be confirmed with prospective study designs.

In conclusion, we present normative values for WLR and PI in infants born preterm and full-term across the EGA range of 26-42 wk. The normative percentile growth curves for WLR and PI provide an indirect but potentially useful measure of visceral adiposity and overall fat mass, respectively. The aggregate results of our chart review study provide a compelling rationale for future studies to validate neonatal WLR with direct measures of visceral fat mass, as has been done in older children and adults. Other critical questions include whether infants born at a lower EGA and/or with greater extent of visceral or total adiposity at birth are destined to have greater adiposity in later years and hence greater cardiometabolic risk and whether such risks can be modified by nutritional strategies to optimize body composition as well as body size. Our results suggest that WLR and PI hold promise as clinically useful, simple, and inexpensive tools as part of a comprehensive assessment of body composition as we progress in our efforts to gain a greater understanding of the fetal and neonatal origins of cardiometabolic sequelae in relation to EGA, body composition at birth, and optimum postnatal nutrition.

\section{METHODS}

\section{Study Population}

We conducted a three-center retrospective cohort study of paired maternal and infant medical records of infants born preterm $(<37 \mathrm{wk})$ and full term $(37-42 \mathrm{wk})$ for a 12 -month period ending 31 August 1998 (1998 epoch) and for a corresponding 12-month period $10 \mathrm{y}$ later ending 31 August 2008 (2008 epoch). Institutional review board approval was received from all three centers before data collection. Parental consent was not required because all data were de-identified during analysis. Study methodology has been previously reported and is now briefly summarized (12).

Infants born preterm. The charts of all inborn infants born preterm and discharged home from the neonatal intensive care unit during the 1998 and 2008 epochs were reviewed. We also included late-preterm inborn infants who were not admitted to the neonatal intensive care unit. Early and late preterm were defined as $<34$ and $34-36$ completed wk gestation, respectively.

Infants born at term. Full term was defined as $37-42$ completed wk gestation. Infants were eligible only if discharged home from the birth hospital. To have approximately three reference full-term infants for each preterm enrollee, we a priori limited our newborn nursery record reviews to the first 25 inborn infants admitted each month for each site and epoch. We included full-term infants born small or large for gestational age. Small for gestational age and large for gestational age were defined as $<10 \%$ and $>90 \%$ for weight, respectively, for gestational age and sex based on recent normative data for infants born in the United States (39).

Exclusion criteria for all births. Exclusion criteria included (i) multiple gestation, (ii) chromosomal disorder or any diagnosed condition affecting infant growth, and (iii) missing maternal chart.

The data collected from the charts of all eligible infants included birth date; sex; EGA at birth based on first-trimester ultrasound if available or on last menstrual period; and birth values for weight $(\mathrm{kg})$, supine length $(\mathrm{cm})$, head circumference $(\mathrm{cm})$, and WC $(\mathrm{cm})$. WC measurements were available from two sites (Bethesda, $\mathrm{MD}$, and Fort Bragg, NC). We calculated BMI $\left(\mathrm{kg} / \mathrm{m}^{2}\right)$ and WLR for all infants with available data. Although weight is typically normalized for standing height in children beyond $2 \mathrm{y}$ of age, we measured supine length in our infants and, therefore, calculated WLR and not WHtR. We also calculated PI $\left(\mathrm{kg} / \mathrm{m}^{3}\right)$. PI is a measure of leanness and is calculated as a relationship between mass and height $(10,11,38)$. It is similar to BMI, but the mass is normalized to the third power of height (or length) rather than the second power, yielding a measure with the same dimensions as density.

Maternal demographic data were also collected, including age at delivery, prepregnancy weight and height, gestational weight gain, pregnancy complications (preeclampsia, gestational hypertension, gestational diabetes), and blood glucose result $(\mathrm{mg} / \mathrm{dl})$ for the routine thirdtrimester 1-h glucose challenge (50-g load). We also recorded marital status, ethnicity, highest level of education completed (high school or less, some college or more), and rank of the active-duty family member identified as the sponsor as enlisted or officer; officer rank requires a college degree. The prepregnancy BMI $\left(\mathrm{kg} / \mathrm{m}^{2}\right)$ was calculated.

\section{Data Analysis}

We validated all recorded data points from the maternal and infant records that were $>3 \mathrm{SD}$ from the mean and also performed a randomly selected audit of $5 \%$ of infant records that further confirmed the accuracy of the recorded data. Maternal characteristics are reported as unadjusted means with SD, or frequency with percent, and were compared between epochs using $\chi^{2}$ tests for categorical data and $t$ tests for continuous data. $Z$ scores for birth weight, length, and head circumference were calculated using means and SDs published by infant sex and EGA (39). Multivariate analysis of variance was used to compare mean infant characteristics between epochs, controlling for infant/maternal demographics and delivery site. Infant demographics included sex and EGA. Maternal demographics included race, prepregnancy BMI, gestational weight gain, preeclampsia, and gestational diabetes. When comparing mean $Z$ scores, EGA and sex were excluded from adjustment. ANOVA results were presented as adjusted means with standard errors. All testing was completed using SAS version 9.0 (SAS Institute, Cary, NC). Results were considered significant at $P<0.05$ (two tailed).

Percentile growth curves for WLR and PI. Sex-specific percentile growth curves for WLR and PI at birth for each EGA week were calculated using the LMS method of Cole and Green (40) as implemented in LMS chart maker version 2.43 software (H. Pan, T.J. Cole, Health for All Children, UK). This method estimates three age-specific parameters: $L$ is the power of the Box-Cox transformation used to achieve a normal distribution for each age group, $M$ is the median value, and $S$ is the coefficient of variation. Curves were fit as cubic splines by nonlinear regression using penalized likelihood. Centile plots, penalized deviance, and generalized Akaike information criterion were used to compare models and select an appropriate degree of smoothing as expressed by the degrees of freedom, and growth curves were fit using the LMS method (40).

EGA- and sex-specific percentiles were obtained from the estimated $L, M$, and $S$ values using the LMS transformation equation:

$$
\text { Percentile }=M(1+L S Z)^{1 / L}
$$

where $Z$ is the $Z$ score corresponding to the given percentile. Similarly, the $Z$ score corresponding to a particular measurement was calculated as

$$
z=\left[(X / M)^{L}-1\right] / L S
$$

where $X$ is the measured value.

Although data were collected on preterm infants starting at EGA of $23 \mathrm{wk}$, the sample size was too small to estimate percentiles separately for males and females born $<30 \mathrm{wk}$ gestation and $<26 \mathrm{wk}$ for males and females combined. 


\section{ACKNOWLEDGMENTS}

We thank Maureen Tate and Russell Moores for their contributions to the early planning and study design. The views expressed in this article are those of the authors and do not necessarily reflect the official policy or position of the Department of the Navy, Army, Department of Defense, or the US government. We are military service members. This work was prepared as part of our official duties. Title 17U.S.C. 105 provides that "Copyright protection under this title is not available for any work of the United States government." Title 17U.S.C. 101 defines a US government work as a work prepared by a military service member or employee of the US government as part of that person's official duties.

\section{STATEMENT OF FINANCIAL SUPPORT}

No financial support was received.

\section{REFERENCES}

1. Fredriks AM, van Buuren S, Fekkes M, Verloove-Vanhorick SP, Wit JM. Are age references for waist circumference, hip circumference and waist-hip ratio in Dutch children useful in clinical practice? Eur J Pediatr 2005; 164:216-22.

2. Bajaj HS, Brennan DM, Hoogwerf BJ, Doshi KB, Kashyap SR. Clinical utility of waist circumference in predicting all-cause mortality in a preventive cardiology clinic population: a PreCIS Database Study. Obesity (Silver Spring) 2009;17:1615-20.

3. Hsieh SD, Muto T. The superiority of waist-to-height ratio as an anthropometric index to evaluate clustering of coronary risk factors among nonobese men and women. Prev Med 2005;40:216-20.

4. Pischon T, Boeing H, Hoffmann K, et al. General and abdominal adiposity and risk of death in Europe. N Engl J Med 2008;359:2105-20.

5. Rodríguez-Rodríguez E, Palmeros-Exsome C, López-Sobaler AM, Ortega RM; Research Group: 920030. Preliminary data on the association between waist circumference and insulin resistance in children without a previous diagnosis. Eur J Pediatr 2011;170:35-43.

6. Elizondo-Montemayor L, Serrano-González M, Ugalde-Casas PA, Bustamante-Careaga H, Cuello-García C. Waist-to-height: cutoff matters in predicting metabolic syndrome in Mexican children. Metab Syndr Relat Disord 2011;9:183-90.

7. Roswall J, Bergman S, Almqvist-Tangen G, et al. Population-based waist circumference and waist-to-height ratio reference values in preschool children. Acta Paediatr 2009;98:1632-6.

8. Catalano PM, Thomas AJ, Avallone DA, Amini SB. Anthropometric estimation of neonatal body composition. Am J Obstet Gynecol 1995;173:1176-81.

9. American Academy of Pediatrics Committee on Nutrition. Nutritional needs of low-birth-weight infants. Pediatrics 1985;75:976-86.

10. Olsen IE, Lawson ML, Meinzen-Derr J, et al. Use of a body proportionality index for growth assessment of preterm infants. J Pediatr 2009;154:48691.

11. Fok TF, Hon KL, Ng PC, et al.; Hong Kong Neonatal Measurements Working Group. Use of anthropometric indices to reveal nutritional status: normative data from 10,226 Chinese neonates. Neonatology 2009;95:23-32.

12. Stokes TA, Holston A, Olsen C, et al. Preterm infants of lower gestational age at birth have greater waist circumference-length ratio and ponderal index at term age than preterm infants of higher gestational ages. J Pediatr 2012;161:735-41.e1.

13. Davidoff MJ, Dias T, Damus K, et al. Changes in the gestational age distribution among U.S. singleton births: impact on rates of late preterm birth, 1992 to 2002. Semin Perinatol 2006;30:8-15.

14. Riddle WR, DonLevy SC. Generating expected growth curves and Z-scores for premature infants. J Perinatol 2010;30:741-50.

15. Vasu V, Modi N. Assessing the impact of preterm nutrition. Early Hum Dev 2007;83:813-8.

16. Katzmarzyk PT, Srinivasan SR, Chen W, Malina RM, Bouchard C, Berenson GS. Body mass index, waist circumference, and clustering of cardiovascular disease risk factors in a biracial sample of children and adolescents. Pediatrics 2004;114:e198-205.

17. Wells JC, Chomtho S, Fewtrell MS. Programming of body composition by early growth and nutrition. Proc Nutr Soc 2007;66:423-34.
18. Ahmad I, Nemet D, Eliakim A, et al. Body composition and its components in preterm and term newborns: a cross-sectional, multimodal investigation. Am J Hum Biol 2010;22:69-75.

19. Micklesfield LK, Evans J, Norris SA, et al. Dual-energy X-ray absorptiometry and anthropometric estimates of visceral fat in Black and White South African Women. Obesity (Silver Spring) 2010;18:619-24.

20. Roggero P, Giannì ML, Amato O, et al. Is term newborn body composition being achieved postnatally in preterm infants? Early Hum Dev 2009;85:349-52.

21. Andersen GS, Girma T, Wells JC, Kæstel P, Michaelsen KF, Friis H. Fat and fat-free mass at birth: air displacement plethysmography measurements on 350 Ethiopian newborns. Pediatr Res 2011;70:501-6.

22. Roggero P, Giannì ML, Amato O, et al. Evaluation of air-displacement plethysmography for body composition assessment in preterm infants. Pediatr Res 2012;72:316-20.

23. Giannì ML, Mora $\mathrm{S}$, Roggero $\mathrm{P}$, et al. Regional fat distribution in children born preterm evaluated at school age. J Pediatr Gastroenterol Nutr 2008;46:232-5.

24. Uthaya S, Thomas EL, Hamilton G, Doré CJ, Bell J, Modi N. Altered adiposity after extremely preterm birth. Pediatr Res 2005;57:211-5.

25. Harrington TA, Thomas EL, Frost G, Modi N, Bell JD. Distribution of adipose tissue in the newborn. Pediatr Res 2004;55:437-41.

26. Després JP, Lemieux I, Bergeron J, et al. Abdominal obesity and the metabolic syndrome: contribution to global cardiometabolic risk. Arterioscler Thromb Vasc Biol 2008;28:1039-49.

27. Phillips LK, Prins JB. The link between abdominal obesity and the metabolic syndrome. Curr Hypertens Rep 2008;10:156-64.

28. Steffes MW, Gross MD, Schreiner PJ, et al. Serum adiponectin in young adults-interactions with central adiposity, circulating levels of glucose, and insulin resistance: the CARDIA study. Ann Epidemiol 2004;14:492-8.

29. Lee JM, Davis MM, Woolford SJ, Gurney JG. Waist circumference percentile thresholds for identifying adolescents with insulin resistance in clinical practice. Pediatr Diabetes 2009;10:336-42.

30. Taylor RW, Williams SM, Grant AM, Ferguson E, Taylor BJ, Goulding A. Waist circumference as a measure of trunk fat mass in children aged 3 to 5 years. Int J Pediatr Obes 2008;3:226-33.

31. Li C, Ford ES, Mokdad AH, Cook S. Recent trends in waist circumference and waist-height ratio among US children and adolescents. Pediatrics 2006;118:e1390-8.

32. Nambiar S, Hughes I, Davies PS. Developing waist-to-height ratio cutoffs to define overweight and obesity in children and adolescents. Public Health Nutr 2010;13:1566-74.

33. Fernández JR, Redden DT, Pietrobelli A, Allison DB. Waist circumference percentiles in nationally representative samples of African-American, European-American, and Mexican-American children and adolescents. J Pediatr 2004;145:439-44.

34. Xiong F, Garnett SP, Cowell CT, et al. Waist circumference and waist-toheight ratio in Han Chinese children living in Chongqing, South-West China. Public Health Nutr 2011;14:20-6.

35. Rubin LP. Postnatal growth in preterm infants: too small, too big, or just right? J Pediatr 2009;154:473-5.

36. Moyer-Mileur LJ, Slater H, Thomson JA, Mihalopoulos N, Byrne J, Varner MW. Newborn adiposity measured by plethysmography is not predicted by late gestation two-dimensional ultrasound measures of fetal growth. J Nutr 2009;139:1772-8.

37. Landmann E, Reiss I, Misselwitz B, Gortner L. Ponderal index for discrimination between symmetric and asymmetric growth restriction: percentiles for neonates from 30 weeks to 43 weeks of gestation. J Matern Fetal Neonatal Med 2006;19:157-60.

38. Lubchenco LO, Hansman C, Boyd E. Intrauterine growth in length and head circumference as estimated from live births at gestational ages from 26 to 42 weeks. Pediatrics 1966;37:403-8.

39. Olsen IE, Groveman SA, Lawson ML, Clark RH, Zemel BS. New intrauterine growth curves based on United States data. Pediatrics 2010;125:e21424.

40. Cole TJ, Green PJ. Smoothing reference centile curves: the LMS method and penalized likelihood. Stat Med 1992;11:1305-19. 